\title{
Stress Birefringence in the Human Cornea
}

\author{
G. P. MISSON* and J. D. STEVENS $\dagger$ \\ Birmingham and London
}

\begin{abstract}
Summary
Stress-induced birefringence is used in engineering to determine the distribution of mechanical stress in experimental models. The phenomenon has previously been reported in the cat cornea but no data for the human exists.

A qualitative description of stress-induced birefringence in the human cornea in vitro is presented. Circularly polarised light is then used to detect the phenomenon in the post-operative human cornea in vivo.
\end{abstract}

The photoelastic effect or stress birefringence is a phenomenon well known in engineering where it has long been used in stress analysis. First described by the physicist Brewster, ${ }^{1}$ transparent substances become birefringent when subjected to mechanical stress. This leads to separation of light into two linearly polarised rays with orthogonal vibration directions and different velocities. A phase difference exists between the two waves resulting in optical interference when the material is observed between crossed polarising filters. This is manifest as the familiar colours of Newton's scale for white light or dark and light bands for monochromatic light.

The distribution of interference phenomena throughout a stressed specimen is related to the distribution of birefringence. This in turn is related to local variations of stress.

The cornea is no different from other transparent materials with respect to the photoelastic effect. Although intrinsically birefringent, ${ }^{2}$ corneal stress birefringence has been shown to occur in the cat. ${ }^{3}$ We are not aware of any similar description for the human or other species.

The work is a preliminary study of stress birefringence in the human cornea. Two related investigations were performed. First, the existence of a phenomenon compatible with the photoelastic effect was confirmed in vitro. Second, corneal birefringence induced by surgical sutures was examined in vivo in the human eye following cataract surgery.

\section{Materials and method}

\section{(1) In vitro}

Three viable (tissue cultured) and three formalin fixed segments of donor corneas were used. Following anchorage of the sclera to a glass microscope slide, a full thickness $8 / 0$ virgin silk suture was placed in clear cornea. Weights were attached to the end of the suture to vary the tension. A range of 0 to $50 \mathrm{~g}$ was used with intervals of $50 \mathrm{mg}$ between 0 and $1 \mathrm{~g}$.

Specimens were observed either with unmodified transmitted white light or with transmitted white light between crossed polarising filters. Photographs were taken at magnifications of between 5 and $15 \times$ using 200 ASA Kodak colour reversal film.

\section{(2) In vivo}

Twenty eyes following cataract surgery were examined. In all cases corneal section had been sutured with interrupted $10 / 0$ monofilament nylon. Photographs were taken at the slit-lamp using a circularly polarising filter in the illumination and observation path. 640ASA 3M tungsten balanced colour reversal film was used. 


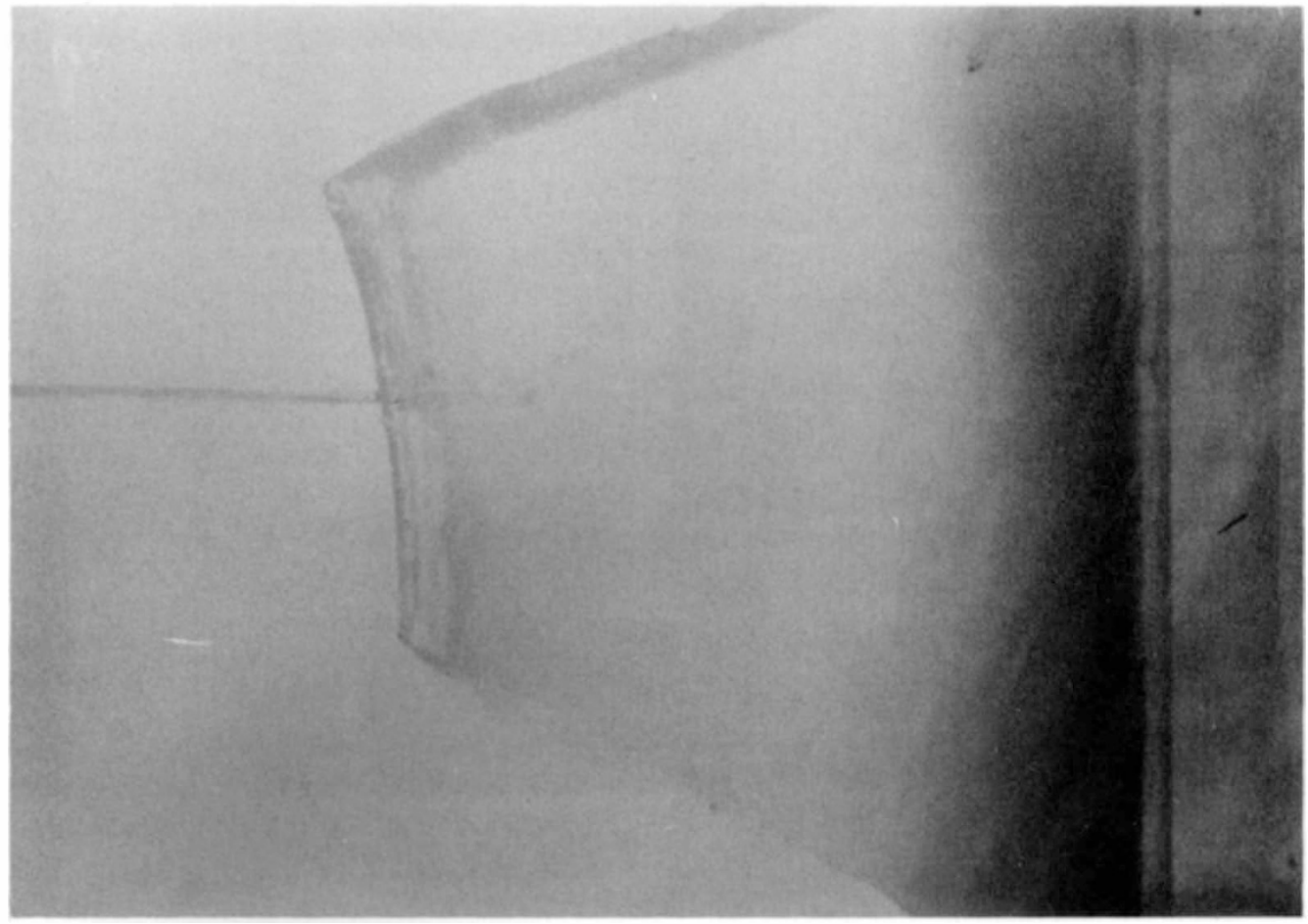

Fig. 1. Corneal segment illuminated with unpolarised light in vitro. No stress applied to suture.

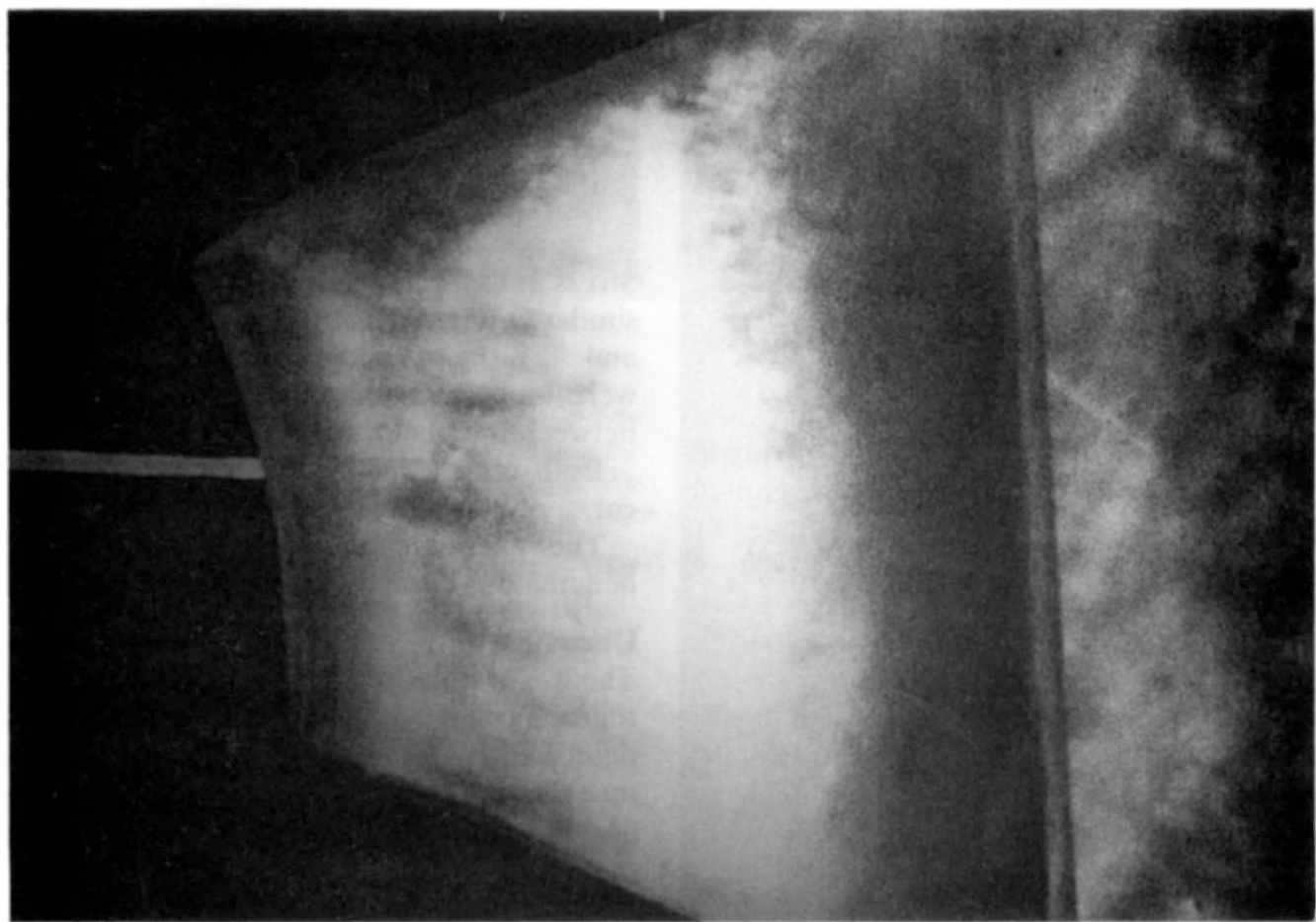

Fig. 2. Corneal segment observed between crossed polarisation filters. No stress applied to suture. 


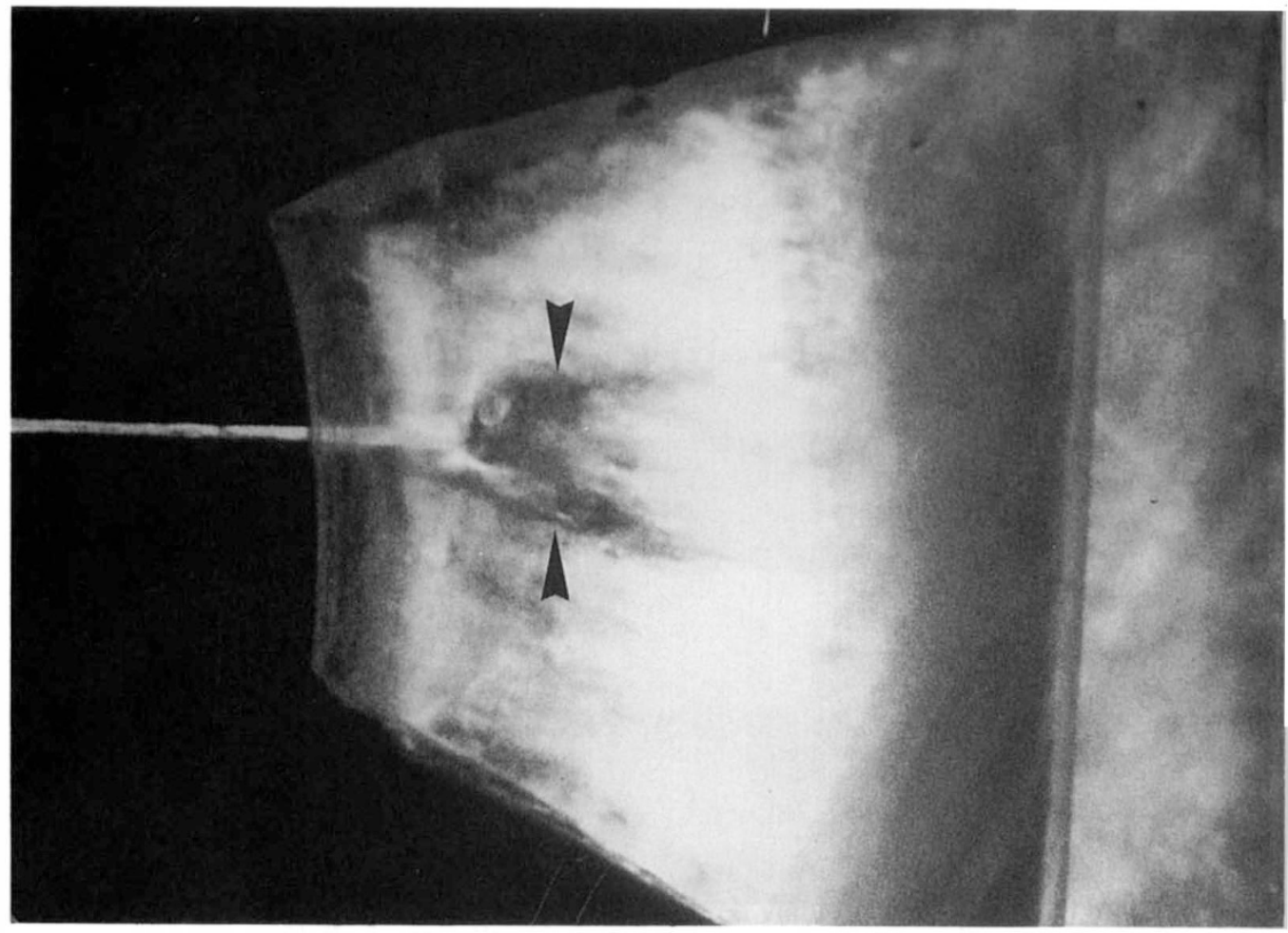

Fig. 3. Corneal segment as in figure 2 now with stress applied to suture. Note V-shaped area of birefringence between arrows.

\section{Results}

\section{(1) In vitro}

With transmitted unpolarised and polarised light, no distinctive features other than background birefringence in the latter were observed when the cornea was unstressed (Figs. 1 and 2).

When traction was applied and the specimen observed between crossed polarising filters (Fig. 3) a dark v-shaped area was noted. The apex occurred at the point of entry of the suture with the limbs extending away from, and at an angle to, the direction of traction. As the force became greater the pattern increased in definition and extent. An end point was reached when any increase in traction produced no further change. In all specimens examined the features were similar.

The amount of tension required to produce an effect with the formalin fixed material was about two orders of magnitude greater than that of the fresh material.
No visible phenomenon was noted in the stressed cornea when observed with unpolarised light.

(2) In vivo

Stress induced by corneal sutures produced a similar pattern to that recorded above (Fig $4 a$ and $4 \mathrm{~b}$ ). In addition, interference colours were noted in the area of compression between the penetrating arms of the sutures.

Both effects resolved when sutures were cut.

The phenomena were poorly visible in oedematous corneas.

\section{Discussion}

The use of polarised light in ophthalmic photography for glare elimination is well described ${ }^{5.6}$ Other uses include assessment of macular integrity ${ }^{7}$ and the investigation of corneal structure. ${ }^{8}$ With the last two exceptions it has been used as an aid to photography rather than as a primary tool for ophthalmic examination. 


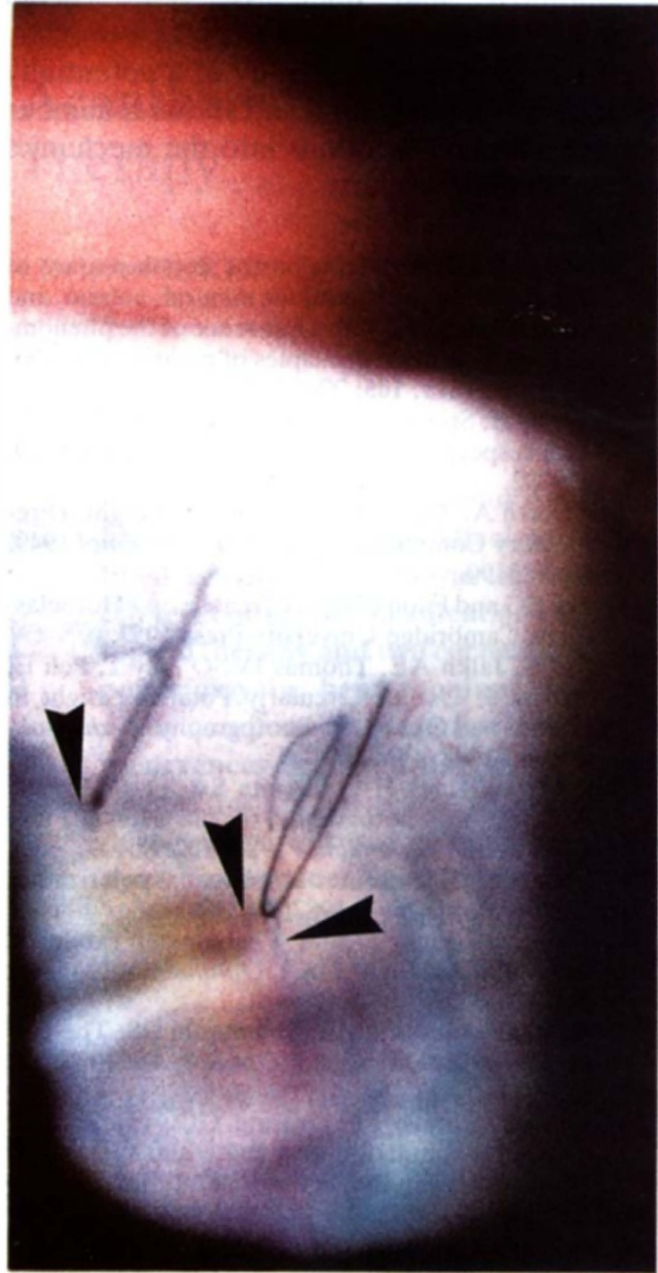

Fig. 4a.

Fig. 4 Stress induced birefringence in vivo at penetrating ends of corneal sutures. Arrow's indicate arms of the $V$-shaped area, the apex of which is at the corneal end of the suture. Taken with circularly polarised light.

It is apparent from the above findings that when subject to mechanical force, be it tension or compression, a change in the optical properties of the human cornea occurs. This is seen by observation between crossed polarising filters. Previous reports of a similar phenomenon in cat corneas was ascribed to the photoelastic effect (stress-induced birefringence). ${ }^{3}$

Tension induced birefringence was noted both in vivo and in vitro occurring at the apex

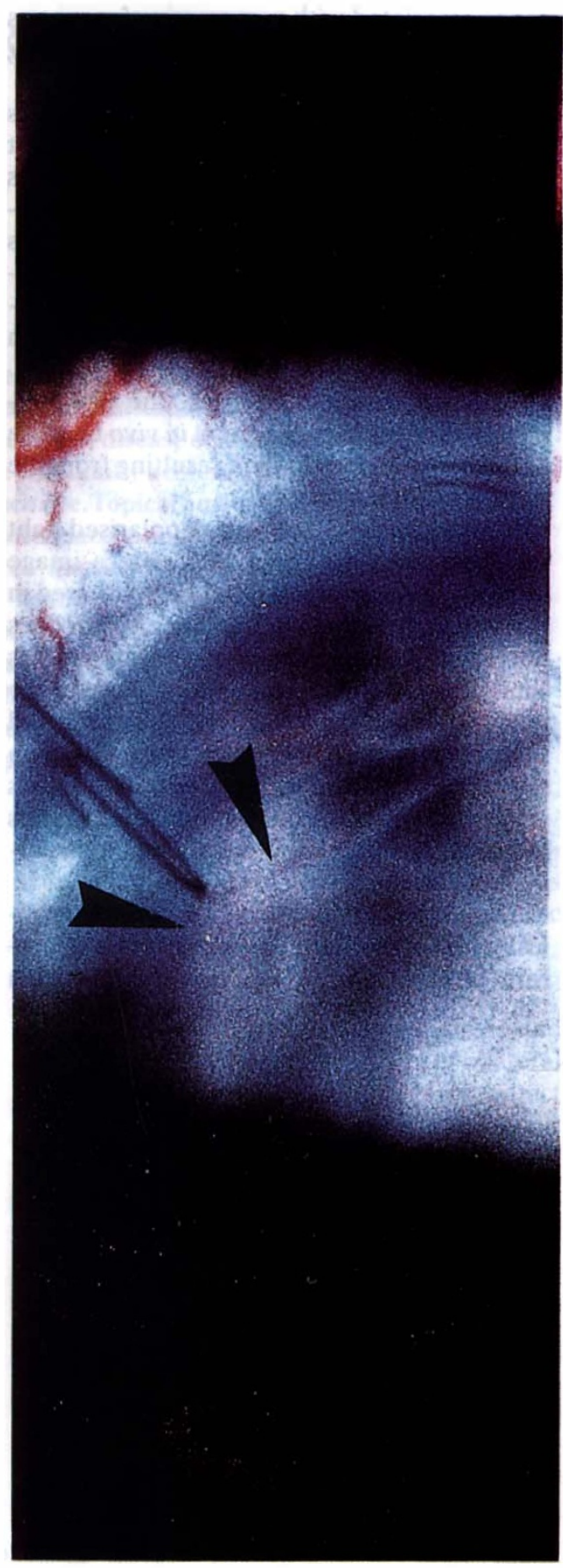

Fig 4b. 
of the corneal arm of the sutures. An interference phenomenon resulting from birefringence associated with compressive forces was observed in vivo between the penetrating arms of sutures.

Without the circularly polarising filter it is possible to see deformation of the cornea at the point of entry of sutures in viva. This is probably due to distortion of collagen lamellae. The use of polarised light enhances this effect and makes visible related stressinduced birefringence which may be considerably more extensive than observation with natural light would suggest.

Although readily observed, the phenomenon is difficult to photograph in vivo because of the reduced illumination resulting from the use of polarising filters.

It might be expected that depolarised light scattered from the iris would reduce image quality. The phenomena has been observed in patients with different iris colour and this does not appear to be the case. This finding is similar to that of previous investigations of the intrinsic birefringence of the cornea ${ }^{8,9}$ and suggests that the phenomena are visible as a result of light scatter within the cornea. This is supported by the observation that stress induced birefringence is poorly visible in corneal oedema.

The cornea is thought to be both mechanically and optically anisotropic. ${ }^{10}$ It is therefore possible that a regional difference in quantitative and qualitative photoelastic properties exists.

This is a preliminary study of a potentially useful clinical technique and allows a number of possible investigations into the mechanics of ophthalmic surgery.

References

${ }^{1}$ Brewster D: Experiments on the depolarisation of light as exhibited by various mineral, animal, and vegetable bodies, with a reference of the phenomena to the general principles of polarisation. Philos Trans 1815, 105: 29-53.

${ }^{2}$ De Vries H, Spoor A, Jielff R: Properties of the eye with respect to polarized light. Physica 1953, 19: 419-32.

${ }^{3}$ Stanworth A: The Cornea in Polarised Light. (Preliminary Communication). Br J Opthalmol 1949, 33: 485-90.

${ }^{4}$ Coker EG and Filon LNG: A Treatise on PHotoelasticity. Cambridge University Press 1957.

${ }^{5}$ Fariza E, Jalkh AE, Thomas JV, O'Day T, Peli E, Acosta J: Use of Circularly Polarized Light in Fundus and Optic Disc Photography. Arch. Ophthalmol 1988, 106: 1001-4.

${ }^{6}$ Sommer A, Kues HA, d'Anna SA, et al: CrossPolarization Photography of the Nerve Fibre Layer. Arch Ophthalmol 1984, 102: 864-9.

${ }^{7}$ Hochheimer BF and Kues HA: Retinal polarization effects. Appl Optics 1982( 21: 3811-18.

${ }^{8}$ Cogan DG: Some Ocular Phenomena Produced with Polarized Light. Arch. Ophthalmol 1941, 25: 391-400.

${ }^{9}$ Cope WT, Wolbrasht ML, Yamanashi BS: The corneal polarization cross. J Opt Soc Am 1978, 68: 1139-41.

${ }^{10}$ Hanna KD, Jouve FE, Waring GO, Ciarlet PG: Computer simulation of arcuate and radial incisions involving the corneoscleral limbus. Eye 1989, 3: 227-39. 